\title{
LIPID METABOLISM DURING WSSV INFECTION IN SHRIMP
}

\author{
Cheng-Shun Cheng, Han-Ching Wang ${ }^{\S}$ \\ Institute of Biotechnology, College of Bioscience and Biotechnology, National Cheng Kung \\ University, Tainan, Taiwan
}

\begin{abstract}
Global metabolic changes in white spot syndrome virus (WSSV)-infected shrimp were recently clarified with proteomics and metabolomics. Interestingly, rerouting of host metabolism (known as Warburg effect in cancer cells) increased availability of energy and biosynthetic building blocks in host cells in shrimp after WSSV infection at the genome replication stage (12 hpi). Moreover, WSSV switched lipid metabolism of host from lipolysis at 12 hpi to lipogenesis at $24 \mathrm{hpi}$ and used it to complete viral replication as well as morphogenesis. Lipolysis was induced by WSSV in hepatopancreas at replication stage (12 hpi), which released free fatty acids that were soon taken up by WSSV target tissues (e.g. hemocytes and stomach). Lipolysis was switched to hemocytes and stomach until lipid in hepatopancreas was exhausted at a late stage of viral infection ( $24 \mathrm{hpi}$ ). Furthermore, beta-oxidation in shrimp may be triggered during WSSV infection. We determined that inhibition of beta-oxidation, or inhibition of fatty acid synthesis (FAS, a key enzyme of lipogenesis), prevented WSSV from completing its replication cycle. Therefore, we inferred that alteration of lipid metabolism might be essential for WSSV virion formation. Our study provided new insights into important changes in host lipid metabolism triggered by an invertebrate virus.
\end{abstract}

\section{KEYWORDS}

Litopenaeus vannamei, White spot syndrome virus, Warburg effect, Lipolysis, Lipogenesis §Corresponding author. TEL: +886-6-2757575 ext 65603-810 FAX: +886-6-276-6505 E-mail: wanghc@mail.ncku.edu.tw 